\title{
Stroke: Physical Fitness, Exercise, and Fatigue
}

\author{
Gillian Mead, ${ }^{1}$ Julie Bernhardt, ${ }^{2}$ and Gert Kwakkel ${ }^{3,4}$ \\ ${ }^{1}$ School of Clinical Sciences and Community Health, Royal Infirmary of Edinburgh, The University of Edinburgh, \\ Little France Crescent, Edinburgh, UK \\ ${ }^{2}$ Stroke Division, Florey Neuroscience Institute, Melbourne, Australia \\ ${ }^{3}$ Department of Rehabilitation Medicine, Researsh Institute MOVE, VU University Medical Centre, \\ 1081 HV Amsterdam, The Netherlands \\ ${ }^{4}$ Rudolf Magnus Institute, University Medical Centre Utrecht, 3508 GA Utrecht, The Netherlands
}

Correspondence should be addressed to Gillian Mead, gmead@staffmail.ed.ac.uk

Received 5 October 2011; Accepted 5 October 2011

Copyright (๑) 2012 Gillian Mead et al. This is an open access article distributed under the Creative Commons Attribution License, which permits unrestricted use, distribution, and reproduction in any medium, provided the original work is properly cited.

If we could put exercise into a drug, it would be one of the most effective medications to prevent vascular disease and treat patients with cardiovascular and cerebrovascular diseases including stroke. Exercise reduces the risk of diabetes and hypertension and improves lipid profiles. There is good evidence that exercise reduces the risk of a first-ever stroke [1]. There is now an increasing body of evidence that suggests that exercise after stroke has a number of benefits and may help address common post-stroke problems including fatigue [2].

This special issue has brought together a series of papers exploring the potential benefits of exercise after stroke, the barriers that exist to increased physical activity after stroke, and the mechanisms and time course of fatigue after stroke. The first paper by D. Deplanque and colleagues shows a dose-response relationship between higher levels of previous leisure time, physical activity, and lower initial stroke severity; this benefit might be mediated by neuroprotective as well as by vascular mechanisms. The low levels of activity seen in stroke survivors (A. Danielsson et al.), even after minor stroke (M. Hildebrand et al.), the decline in cardiorespiratory fitness ("Reduced cardiorespiratory fitness after stroke: biological consequences exercise-induced adaptations" by $\mathrm{S}$. A. Billinger et al. and "Cardiopulmonary response to exercise testing in people with chronic stroke: a retrospective study" by S. A. Billinger et al.), the relationship between walking endurance and gait stability (M. Iosa et al.), and the potentially protective effects of exercise on bone loss (K. Borschmann) and falls risk (H. van Duijnhoven et al.) all underline the importance of physical fitness training as a key target in the multidisciplinary management of organized stroke care in the first weeks after stroke and beyond. Early commencement of physical activity is feasible after stroke [3] and appears to fast track recovery of walking [4]. Physical fitness training after stroke improves physical function including walking [5].

Observational studies of stroke patients continue to show that most patients are inactive during their stay in hospitals (T. West and J. Bernhardt) in both acute and rehabilitation wards, suggesting that the physical fitness of stroke patients is still a neglected part of the agenda in stroke management. We would urge therapists to consider how to build fitness training into their regime in addition to focusing on improving function after stroke. As therapists' time is often limited, we need to consider novel, more time-efficient methods to increase physical activity such as fitness classes, circuit class training, family mediated exercises, and telerehabilitation services [6]. For example, a recent Cochrane review of English and Hillier [7] has shown that task-oriented circuit class training is effective in terms of gait speed, walking distance, and balance control. A large randomised controlled trial is underway in which the cost effectiveness of circuit class training as a replacement for usual care is compared, acknowledging that lack of time and lack of staff are major barriers to the implementation of intensive practice after stroke [8].

If we are to optimize stroke survivors' participation in physical activity, it is important to understand the factors determining participation. M. A. Alzaharini et al., in their paper "Mood and balance are associated with free-living 
physical activity of people after stroke residing in the community," reported that mood and balance are important predictors of physical activity. The existing literature suggests that self-efficacy, physical activity beliefs, and social support are important determinants of exercise behaviour after stroke (J. Morris et al.). G. Banks and colleagues, in their paper "Exercise differences are different after stroke," report that exercise preferences after stroke differ from those people without stroke, whilst Tiedemann and colleagues, in "Predictors of adherence to a structured exercise program and physical activity participation in community dwellers after stroke," have provided new insights into the factors predicting adherence to group exercises. Taken together, it would seem sensible to ensure that exercise beliefs and preferences are taken into account when advising stroke survivors to be more physically active. Stroke services are now beginning to explore how to establish community-based exercise environments for stroke survivors which will, one hopes, help maintain or improve fitness after usual stroke rehabilitation has been completed [2].

From the patient's perception, post-stroke fatigue often seems to be triggered at the time of stroke (perhaps by the brain lesion) and that exercise, rehabilitation, and good sleep may reduce feelings of fatigue (V. L. Barbour and G. E. Mead). These observations might help provide new targets for the development of interventions for post-stroke fatigue. We need new treatments for fatigue because it seems to persist in the long term (A. Lerdal et al.). The initial level of fatigue is the main determinant of increasing fatigue over time (H. M. van Eijsden et al.), and so targeting fatigue soon after stroke may be important to prevent it from persisting. Post-stroke fatigue is a well-recognized symptom that should be differentiated from often accompanying symptoms such as depression. There is one published case definition of clinically significant post-stroke fatigue [9], but no clear consensus about which of the plethora of outcome measures recommending different cutoffs for determining fatigue that we should be using. This is an area where further research is needed.

Unfortunately, we have no evidence-based interventions available to prevent and treat the post-stroke fatigue. The treatment of this multidimensional symptom will probably require a multidisciplinary approach that targets the physical as well as cognitive aspects of fatigue. However, the underlying mechanisms that cause fatigue as well as the impact of physical (in) activity and physical fitness on this time course are poorly understood and still in their infancy. The present special issue focused on "Stroke: Physical Fitness, Exercise, and Fatigue" is an important step forward in our journey aiming at preventing inactivity and reducing fatigue.

Gillian Mead Julie Bernhardt Gert Kwakkel
[2] G. E. Mead and J. Bernhardt, "Physical fitness training after stroke, time to implement what we know: more research is needed," International Journal of Stroke, vol. 6, no. 6, pp. 506508, 2011.

[3] J. Bernhardt, H. Dewey, A. Thrift, J. Collier, and G. Donnan, "A very early rehabilitation trial for stroke (AVERT): phase II safety and feasibility," Stroke, vol. 39, no. 2, pp. 390-396, 2008.

[4] T. B. Cumming, A. G. Thrift, J. M. Collier et al., "Very early mobilization after stroke fast-tracks return to walking: further results from the phase II AVERT randomized controlled trial," Stroke, vol. 42, no. 1, pp. 153-158, 2011.

[5] M. Brazzelli, D. H. Saunders, C. A. Greig, and G. E. Mead, "Physical fitness training for stroke patients," Cochrane Database of Systematic Reviews, no. 11, 2011.

[6] P. Langhorne, J. Bernhardt, and G. Kwakkel, "Stroke rehabilitation,” The Lancet, vol. 377, no. 9778, pp. 1693-1702, 2011.

[7] C. English and S. L. Hillier, "Circuit class therapy for improving mobility after stroke," Cochrane Database of Systematic Reviews, vol. 7, Article ID CD007513, 2010.

[8] I. G. van de Port, L. Wevers, H. Roelse, L. van Kats, E. Lindeman, and G. Kwakkel, "Cost-effectiveness of a structured progressive task-oriented circuit class training programme to enhance walking competency after stroke: the protocol of the FITStroke trial," BMC Neurology, vol. 9, article 43, 2009.

[9] J. Lynch, G. E. Mead, C. A. Greig, A. Young, S. Lewis, and M. Sharpe, "Fatigue after stroke: the development and evaluation of a case definition," Journal of Psychosomatic Research, vol. 63, no. 5, pp. 539-544, 2007.

\section{References}

[1] C. D. Lee, A. R. Folsom, and S. N. Blair, "Physical activity and stroke risk: a meta-analysis," Stroke, vol. 34, no. 10, pp. 2475 2481, 2003. 


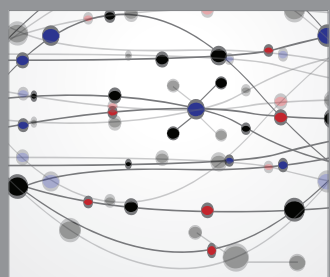

The Scientific World Journal
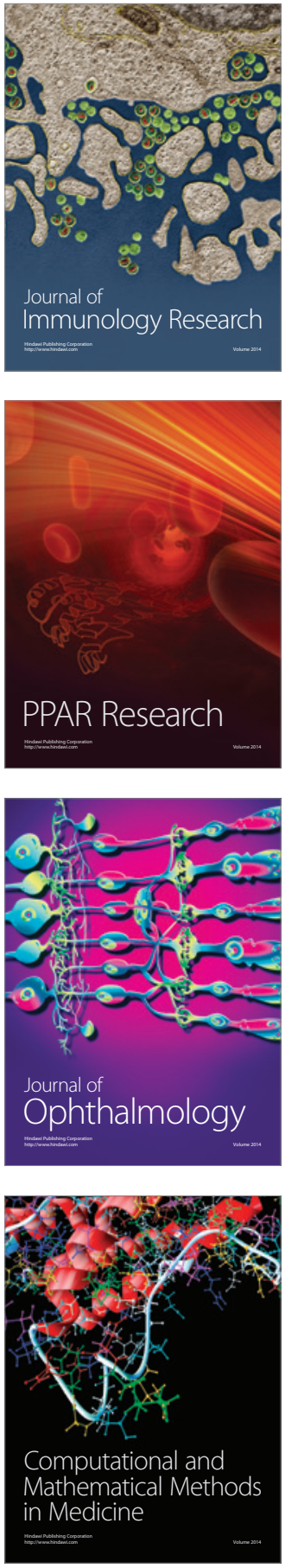

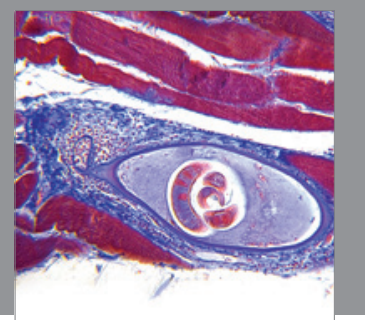

Gastroenterology

Research and Practice
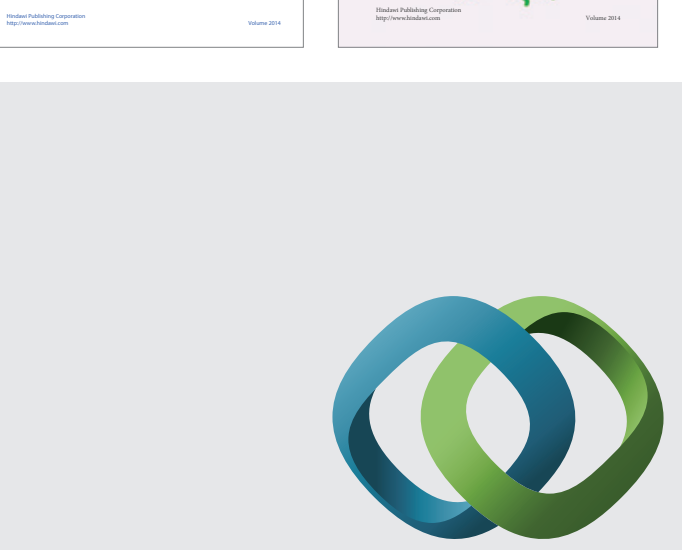

\section{Hindawi}

Submit your manuscripts at

http://www.hindawi.com
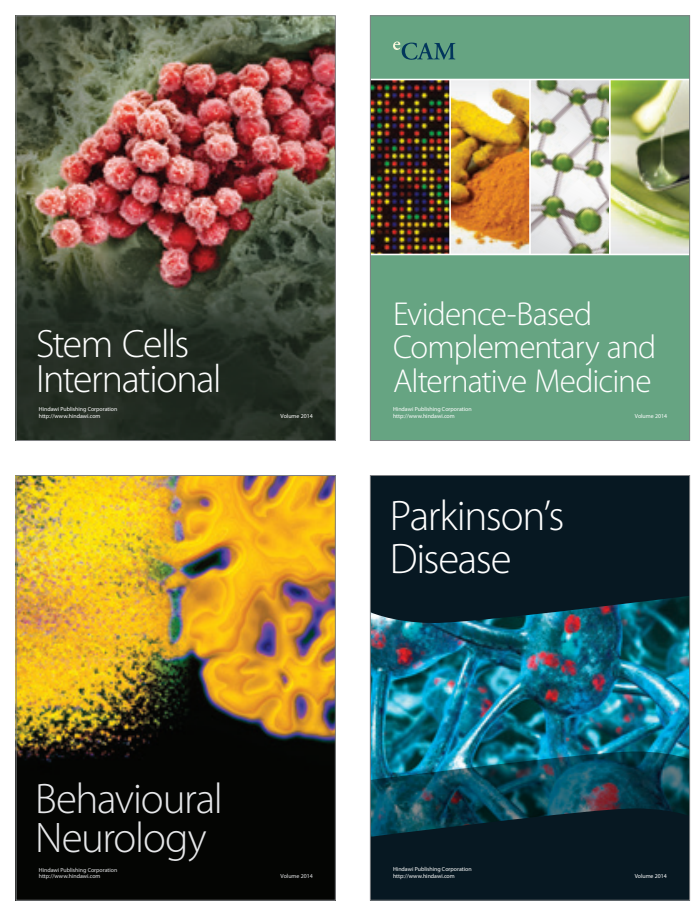

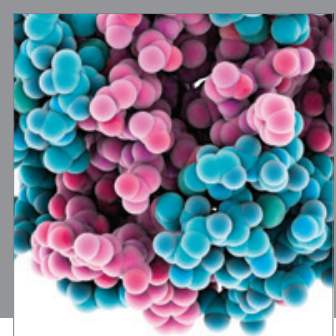

Journal of
Diabetes Research

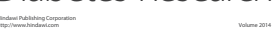

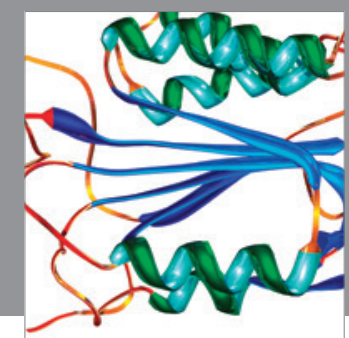

Disease Markers
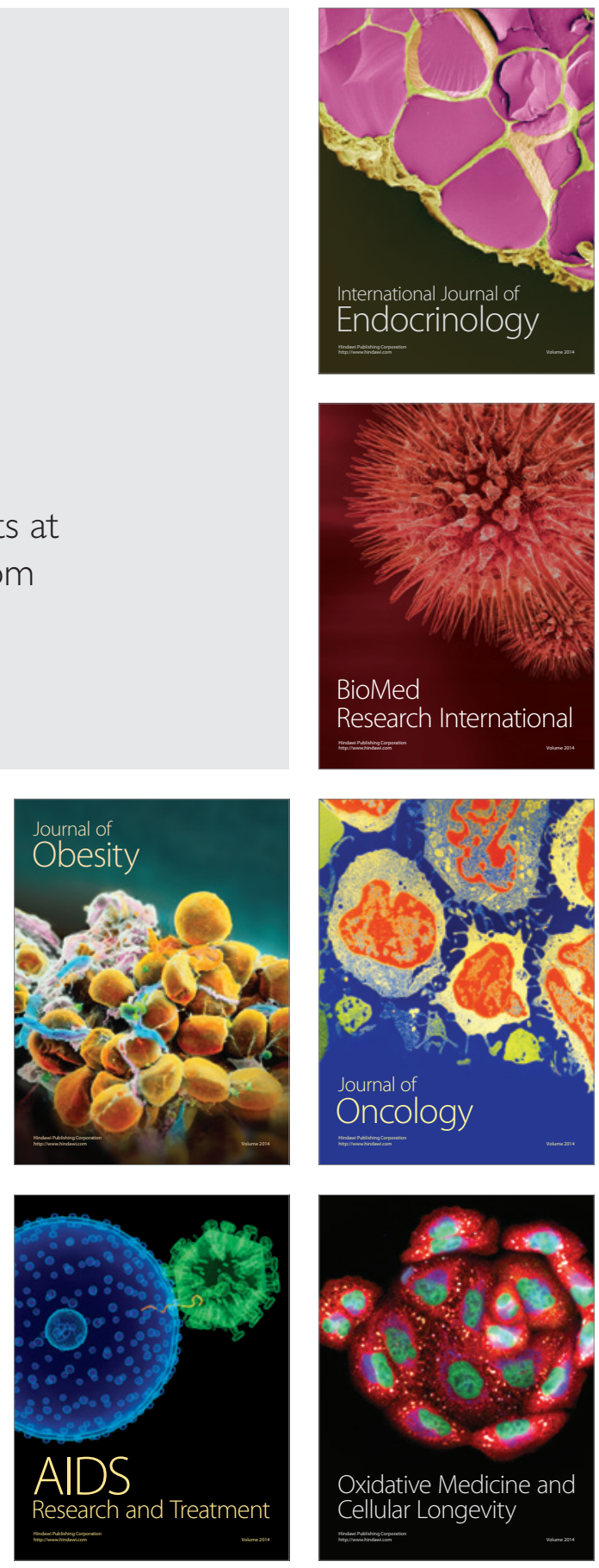